\title{
Symmetry breaking, mixing, instability, and low frequency variability in a minimal Lorenz-like system
}

Article

Accepted Version

Lucarini, V. and Fraedrich, K. (2009) Symmetry breaking, mixing, instability, and low frequency variability in a minimal Lorenz-like system. Physical Review E, 80 (2). 026313. ISSN 1539-3755 doi: https://doi.org/10.1103/PhysRevE.80.026313 Available at https://centaur.reading.ac.uk/27134/

It is advisable to refer to the publisher's version if you intend to cite from the work. See Guidance on citing.

To link to this article DOI: http://dx.doi.org/10.1103/PhysRevE.80.026313

Publisher: American Physical Society

All outputs in CentAUR are protected by Intellectual Property Rights law, including copyright law. Copyright and IPR is retained by the creators or other copyright holders. Terms and conditions for use of this material are defined in the End User Agreement.

www.reading.ac.uk/centaur 
Central Archive at the University of Reading

Reading's research outputs online 


\title{
Symmetry breaking, mixing, instability, and low-frequency variability in a minimal Lorenz-like system
}

\author{
Valerio Lucarini* \\ Department of Mathematics, University of Reading, Whiteknights, P.O. Box 220, Reading RG6 6AX, United Kingdom, \\ Department of Meteorology, University of Reading, Earley Gate, P.O. Box 243, Reading RG6 6BB, United Kingdom, \\ and Department of Physics, University of Bologna, Viale Berti-Pichat 6/2, 40127 Bologna, Italy \\ Klaus Fraedrich \\ Meteorologisches Institut, KlimaCampus, University of Hamburg, Grindelberg 5, 20144 Hamburg, Germany
}

(Received 5 December 2008; published 21 August 2009)

\begin{abstract}
Starting from the classical Saltzman two-dimensional convection equations, we derive via a severe spectral truncation a minimal 10 ODE system which includes the thermal effect of viscous dissipation. Neglecting this process leads to a dynamical system which includes a decoupled generalized Lorenz system. The consideration of this process breaks an important symmetry and couples the dynamics of fast and slow variables, with the ensuing modifications to the structural properties of the attractor and of the spectral features. When the relevant nondimensional number (Eckert number Ec) is different from zero, an additional time scale of $O\left(\mathrm{Ec}^{-1}\right)$ is introduced in the system, as shown with standard multiscale analysis and made clear by several numerical evidences. Moreover, the system is ergodic and hyperbolic, the slow variables feature long-term memory with $1 / f^{3 / 2}$ power spectra, and the fast variables feature amplitude modulation. Increasing the strength of the thermal-viscous feedback has a stabilizing effect, as both the metric entropy and the Kaplan-Yorke attractor dimension decrease monotonically with Ec. The analyzed system features very rich dynamics: it overcomes some of the limitations of the Lorenz system and might have prototypical value in relevant processes in complex systems dynamics, such as the interaction between slow and fast variables, the presence of long-term memory, and the associated extreme value statistics. This analysis shows how neglecting the coupling of slow and fast variables only on the basis of scale analysis can be catastrophic. In fact, this leads to spurious invariances that affect essential dynamical properties (ergodicity, hyperbolicity) and that cause the model losing ability in describing intrinsically multiscale processes.
\end{abstract}

DOI: 10.1103/PhysRevE.80.026313

PACS number(s): 47.20.Ky, 05.45.- a, 47.10.Fg, 47.52.+j

\section{INTRODUCTION}

The Lorenz system [1] has a central role in modern science as it has provided the first example of low-dimensional chaos [2], and has literally paved the way for new scientific paradigms. The Lorenz system can be derived with a minimal truncation of the Fourier-modes projection of the twodimensional (2D) Boussinesq convection equations introduced by Saltzman [3], where a specific selection of the spatial symmetry of the fields is considered. Extensions of the Lorenz system taking into account higher-order spectral truncations in the 2D case have been presented (see, e.g., [4-6]) whereas in [7] the standard procedure has been extended to the three-dimensional (3D) case.

The mathematical properties of the Lorenz system have been the subject of an intense analysis, which has addressed the bifurcations as well as the characteristics of the strange attractors realized within certain parametric ranges. In particular, we wish to mention the investigation of Feigenbaum sequences of bifurcations [8] and the study of the properties of its attractor via the analysis of unstable periodic orbits [9]. For several classic results, see [10]. Recently, at more theoretical level, the investigation of Lorenz-like systems has stimulated the introduction of the family of singular hyper-

\footnotetext{
*v.lucarini@reading.ac.uk
}

bolic systems as extension of the family of hyperbolic systems [11]. Moreover, moving from the theory developed for nonequilibrium systems [12] starting from the Ruelle response theory $[13,14]$, in a recent paper a careful verification on the Lorenz system of the Kramers-Kronig dispersion relations and sum rules has been performed [15].

In spite of its immense value, the Lorenz system does not provide an efficient representation of several crucial phenomena typically associated with complex chaotic systems. When looking at finite time predictability properties, the Lorenz system features an unrealistic return-of-skill in the forecast, i.e., there are regions in the attractor within which all infinitesimal uncertainties decrease with time [16]. Additionally, the Lorenz system does not feature an interplay between fast and slow variables, so that it cannot mimic the coupling between systems with different internal time scales. Palmer [17] introduced artificially ad hoc components to the Lorenz system in order to derive a toy-model able to generate lowfrequency variability. Moreover, as discussed in [18], the Lorenz system does not allow for a closed-form computation of the entropy production.

Some pathologies emerge also when looking at the relationship between the Lorenz system and higher-order spectral truncation of the Saltzman equations. Recently, Chen and Price showed that the Lorenz system is a specific member of a class of equivalence of systems whose dynamics is invariant with respect to a symmetry transformation [19]. 
In this work, we wish to propose a minimal dynamical system, possibly with some paradigmatic value, able to overcome some of the limitation of the Lorenz system, and endowed with a much richer dynamics. Starting from the $2 \mathrm{D}$ convection equations, we derive with a truncation à la Lorenz a minimal 10 degrees of freedom (d.o.f.) ordinary differential equations (ODEs) system which includes the description of the thermal effect of viscous dissipation. We discuss how, while neglecting this process lead us to a dynamical system which includes a decoupled (generalized) Lorenz system, its consideration breaks an important symmetry of the systems, couples the dynamics of fast and slow variable, with the ensuing modifications to the structural properties of the attractor and in the spectral features of the system. Numerical results are provided for a specific selection of parameters' values based upon the classical Lorenz choice.

\section{RAYLEIGH-BENARD CONVECTIVE SYSTEM}

We consider a thermodynamic fluid having kinematic viscosity $\nu$, thermal-conductivity $k$, thermal capacity at constant volume $C_{v}$, and linearized equation-of-state $\rho=\rho_{0}(1-\alpha T)$. The 2D $(x, z)$ Rayleigh-Benard top-heavy convective system confined within $z \in[0, H]$ can be completely described, when adopting the Boussinesq approximation, by the following system of partial differential equations (PDEs) :

$$
\begin{gathered}
\partial_{t} \nabla^{2} \psi+J\left(\psi, \nabla^{2} \psi\right)=g \alpha \partial_{x} \theta+\nu \nabla^{4} \psi \\
\partial_{t} \theta+J(\psi, \theta)=\frac{\Delta T}{H} \partial_{x} \psi+k \nabla^{2} \theta+\frac{\nu}{C_{v}} \partial_{i j} \psi \partial_{i j} \psi
\end{gathered}
$$

where $g$ is the gravity acceleration, $\vec{v}=(u, w)=\left(-\partial_{z} \psi, \partial_{x} \psi\right)$, $T=T_{0}-z \Delta T / H+\theta$, with $H$ uniform depth of the fluid and $\Delta T$ imposed temperature difference. The suitable boundary conditions in the case of free-slip system are $\theta(x, z=0)=\theta(x, z$ $=H)=0$ and $\psi(x, z=0)=\psi(x, z=H)=\nabla^{2} \psi(x, z=0)=\nabla^{2} \psi(x, z$ $=H)=0$ for all values of $x$. These PDEs can be nondimensionalized by applying the linear transformations $x=H \hat{x}, z$ $=H \hat{z}, t=H^{2} / k \hat{t}, \psi=k \hat{\psi}$, and $\theta=k \nu /\left(g \alpha H^{3}\right) \hat{\theta}$ :

$$
\begin{gathered}
\partial_{\hat{t}} \hat{\nabla}^{2} \hat{\psi}+J\left(\hat{\psi}, \hat{\nabla}^{2} \hat{\psi}\right)=\sigma \partial_{\hat{x}} \hat{\theta}+\sigma \hat{\nabla}^{4} \hat{\psi}, \\
\partial_{\hat{t}} \hat{\theta}+J(\hat{\psi}, \hat{\theta})=R \partial_{\hat{x}} \hat{\psi}+\hat{\nabla}^{2} \hat{\theta}+\sigma \operatorname{Ec} \partial_{i j} \hat{\psi} \partial_{i j} \hat{\psi},
\end{gathered}
$$

where $\sigma=\nu / k$ is the Prandl number, $R=g \alpha H^{3} \Delta T /(k \nu)$ is the Rayleigh number, and $\mathrm{Ec}=k^{2} /\left(C_{v} \Delta T H^{2}\right)$ is the Eckert number, and the suitable boundary conditions are $\hat{\theta}(\hat{x}, \hat{z}=0)$ $=\hat{\theta}(\hat{x}, \hat{z}=1)=0 \quad$ and $\quad \hat{\psi}(\hat{x}, \hat{z}=0)=\hat{\psi}(\hat{x}, \hat{z}=1)=\hat{\nabla}^{2} \hat{\psi}(\hat{x}, \hat{z}=0)$ $=\hat{\nabla}^{2} \hat{\psi}(\hat{x}, \hat{z}=1)=0$. The Eckert number in Eq. (2b) quantifies the impact of viscous dissipation on the thermal balance of the system. As this number is usually rather small in actual fluids, the corresponding term in the two previous sets of PDEs is typically discarded. Following the strategy envisioned by Saltzman [3] and Lorenz [1], we perform a truncated Fourier expansion of $\hat{\psi}$ and $\hat{\theta}$, assuming that they are periodic along the $\hat{x}$ and $\hat{z}$ with periodicity of $2 / a$ and 2 , respectively. When boundary conditions are considered, we then derive a set of ODEs describing the temporal evolution of the corresponding (complex-valued) modes $\Psi_{m, n}$ and $\Theta_{m, n}$, which are associated with the wavevector $\vec{K}$ $=(\pi a n, \pi m)$. See [3] for a detailed derivation in the case $\mathrm{Ec}=0$, considering that the sign of the term proportional to $R$ is wrong in both Eqs. 34 and 35. In the case $\mathrm{Ec}=0$, the seminal Lorenz system can be derived by severely truncating the system, considering the evolution equation for the real part of $\Psi_{1,1}$ and for the imaginary parts of $\Theta_{1,1}$ and $\Theta_{0,2}$, and performing suitable rescaling (see below). While $\Theta_{0,2}$ has no real part because of the boundary conditions [3], neglecting the imaginary (real) part of $\Psi_{1,1}\left(\Theta_{1,1}\right)$ amounts to an arbitrary selection of the phase of the waves in the system. An entire hierarchy of generalized Lorenz models, all obeying to this constraint, can be derived with lengthy but straightforward calculations. See, e.g., [4-6] for detailed discussion of these models.

\section{SYMMETRIES OF THE EXTENDED LORENZ SYSTEM}

In this work we include the modes $\Psi_{1,1}, \Psi_{2,2}, \Theta_{1,1}$, and $\Theta_{2,2}$ in our truncation, and retain both the real and the imaginary parts, whereas the considered horizontally symmetric modes $\Theta_{0,2}$ and $\Theta_{0,4}$ are, as mentioned above, imaginary. Finally, we assume, in general, a nonvanishing value for Ec. If we define $\Psi_{1,1}=\alpha\left(X_{1}+i X_{2}\right), \quad \Theta_{1,1}=\beta\left(Y_{1}+i Y_{2}\right), \quad \Psi_{2,2}$ $=\alpha\left(A_{1}+i A_{2}\right), \Theta_{2,2}=\beta\left(B_{1}+i B_{2}\right), \Theta_{0,2}=i \gamma Z_{1}$, and $\Theta_{0,4}=i \gamma Z_{2}$, with $\alpha=\left(a^{2}+1\right) /(2 \sqrt{2} a), \quad \beta=\pi^{3}\left(a^{2}+1\right)^{3} /\left(2 \sqrt{2} a^{2}\right)$, and $\gamma$ $=\pi^{3}\left(a^{2}+1\right)^{3} /\left(2 a^{2}\right)$, set $a=1 / \sqrt{2}$ [1], we derive the following system of real ODEs:

$$
\begin{gathered}
\dot{X}_{1}=\sigma Y_{2}-\sigma X_{1} \\
\dot{X}_{2}=-\sigma Y_{1}-\sigma X_{2} \\
\dot{Y}_{1}=X_{2} Z_{1}-r X_{2}-Y_{1}+5 /(\sqrt{2} \pi) \sigma \operatorname{Ec}\left(A_{1} X_{1}+A_{2} X_{2}\right) \\
\dot{Y}_{2}=-X_{1} Z_{1}+r X_{1}-Y_{2}+5 /(\sqrt{2} \pi) \sigma \operatorname{Ec}\left(A_{2} X_{1}-A_{1} X_{2}\right)
\end{gathered}
$$

$$
\dot{A}_{1}=\sigma / 2 B_{2}-4 \sigma A_{1}
$$$$
\dot{A}_{2}=-\sigma / 2 B_{1}-4 \sigma A_{2}
$$$$
\dot{B}_{1}=4 A_{2} Z_{2}-2 r A_{2}-4 B_{1}-1 /(2 \sqrt{2} \pi) \sigma \mathrm{Ec}\left(X_{1}^{2}-X_{2}^{2}\right)
$$$$
\dot{B}_{2}=-4 A_{1} Z_{2}+2 r A_{1}-4 B_{2}-1 /(\sqrt{2} \pi) \sigma \mathrm{Ec} X_{1} X_{2}
$$$$
\dot{Z}_{1}=X_{1} Y_{2}-X_{2} Y_{1}-b Z_{1}
$$$$
\dot{Z}_{2}=4 A_{1} B_{2}-4 A_{2} B_{1}-4 b Z_{2},
$$

where the dot indicates the derivative with respect to $\tau$ $=\pi^{2}\left(a^{2}+1\right) t=3 / 2 \pi^{2} t, \quad r=R / R_{c}=R /\left(27 \pi^{4} / 4\right)$ is the relative 
Rayleigh number, and $b$ is a geometric factor. Note that if we exclude the faster varying modes $\Psi_{2,2}$ and $\Theta_{2,2}$ in our truncation (as in the Lorenz case) the Eckert number is immaterial in the equations of motion, the basic reason being that viscous dissipation acts on small spatial scales. Therefore, the ODEs (3a)-(3j) provide the minimal system which includes the feedback due to the thermal effect of viscosity.

If we retain all the variables in the system $(3 \mathrm{a})-(3 \mathrm{j})$ and Ec is set to 0 , the following symmetry is obeyed. Let $S\left(t, S_{0}\right)=[X(t), Y(t), A(t), B(t), Z(t)]^{T}$ — with

$\bullet(t)=\left[\bullet_{1}(t), \bullet_{2}(t)\right]$ - be a solution of the system with initial conditions $S_{0}=[X(0), Y(0), A(0), B(0), Z(0)]^{T}$. We then have that

$$
T^{-1}(\omega, \phi) S\left[t, T(\omega, \phi) S_{0}\right]=S\left(t, S_{0}\right)
$$

for all $(\omega, \phi) \in \mathfrak{R}^{2}$, where $T(\omega, \phi)$ is a real linear orthogonal $\left[T^{-1}(\omega, \phi)=T^{T}(\omega, \phi)\right]$ transformation

$$
T(\omega, \phi) S=\left(\begin{array}{ccccc}
R_{\omega} & 0 & 0 & 0 & 0 \\
0 & R_{\omega} & 0 & 0 & 0 \\
0 & 0 & R_{\phi} & 0 & 0 \\
0 & 0 & 0 & R_{\phi} & 0 \\
0 & 0 & 0 & 0 & I
\end{array}\right)\left(\begin{array}{c}
X \\
Y \\
A \\
B \\
Z
\end{array}\right),
$$

with $I$ being the $2 \mathrm{X} 2$ identity matrix and $R_{\xi}$ being the $2 \mathrm{X} 2$ rotation matrix of angle $\xi$. The $T(\omega, \phi)$-matrix shifts the phases of both the $\Psi_{1,1}$ and $\Theta_{1,1}$ waves by of the same angle $\omega$ and, independently, the phases of both the $\Psi_{2,2}$ and $\Theta_{2,2}$ waves by the same angle $\phi$. The symmetry [Eqs. (4) and (5)] gives a solid framework to and generalizes the results by Chen and Price [19].

\section{STATISTICAL PROPERTIES OF THE SYSTEM- SYMMETRIC CASE}

In the $\mathrm{Ec}=0$ case, we expect the presence of degeneracies in the dynamics, leading to nonergodicity of the system. Since, from Eq. (4), we have that $S\left[t, T(\omega, \phi) S_{0}\right]$ $=T(\omega, \phi) S\left(t, S_{0}\right)$, we readily obtain that the statistical properties of the flow depend on the initial conditions, and that by suitably choosing the matrix $T(\omega, \phi)$, we can, e.g., exchange the statistical properties of $X_{1}$ and $Y_{1}$ with those of $X_{2}$ and $Y_{2}$ by setting $\omega=\pi / 2$ (and similarly for the $A$ and $B$ variables by setting $\phi=\pi / 2$ ). Whereas, in actual integration, numerical noise coupled with sensitive dependence on initial conditions (see below) breaks the instantaneous identity $S\left[t, T(\omega, \phi) S_{0}\right]=T(\omega, \phi) S\left(t, S_{0}\right)$ after sufficiently long time, the statistical properties of the observables transform according to the symmetry defined in Eq. (5).

The dynamics of the variables $\left(X_{1}, X_{2}, Y_{1}, Y_{2}, Z_{1}\right)$ defines an extended Lorenz system [19]. The classical threecomponent system results from a specific selection of the phase of the waves of the system, which is obtained by setting vanishing initial conditions for $X_{2}$ and $Y_{1}$. With such a choice, we have that $X_{2}=Y_{1}=0$ at all times, whereas the $x$ and $y$ variables of the Lorenz system are $X_{1}$ and $Y_{2}$, respectively. As a consequence of the symmetry given in Eqs. (4) and (5), the statistical properties of $Z_{1}$ do not depend on the initial conditions, and agree with those of the $z$ variable of the classical Lorenz system. Moreover, the statistical properties of the quadratic quantities $X^{2} \equiv X_{1}^{2}+X_{2}^{2}, Y^{2} \equiv Y_{1}^{2}+Y_{2}^{2}$, and $X Y \equiv X_{1} Y_{2}-X_{2} Y_{1}$ do not depend on the initial conditions (whereas those of each term in the previous sums do), and agree (for all values of $r, \sigma, b$ ) with those of $x^{2}$ and $y^{2}$, and $x y$ of the classical Lorenz system, respectively. Analogously, since the symmetry defined in Eq. (5) is obeyed, the statistical properties of $A_{1}, A_{2}, B_{1}, B_{2}$ depend on the initial conditions, whereas the statistical properties of the quadratic quantities $A^{2} \equiv A_{1}^{2}+A_{2}^{2} B^{2} \equiv B_{1}^{2}+B_{2}^{2}, A B \equiv A_{1} B_{2}-A_{2} B_{1}$, and of $Z_{2}$ are well-defined. The symmetry [Eqs. (4) and (5)] implies that the attractor of the system (3a)-(3j) can be expressed as a Cartesian product of a two-torus times a "fundamental" attractor. The initial conditions will define where on the torus the system is located.

From a physical point of view, the symmetry [Eqs. (4) and (5)] is related to the fact that the system does not preferentially select streamfunction and temperature waves of a specific phase, and, does not mix phases. Therefore, in a statistical sense, the relative strength of waves with the same periodicity but different phase can be arbitrarily chosen by suitably selecting the initial conditions. Instead, the statistical properties of the space-averaged convective heat transport, which is determined by a linear combination of $Z_{1}$ and $Z_{2}$, as well as those of the total kinetic energy (determined by a linear combination of $X^{2}$ and $A^{2}$ ) and available potential energy (determined by a linear combination of $Y^{2}$ and $B^{2}$ ) of the fluid [3] must not depend on the initial conditions-but only on the system's parameters - as they are robust (thermo-)dynamical properties of the flow.

In general, since the dynamics of the variables $\left(X_{1}, X_{2}, Y_{1}, Y_{2}, Z_{1}\right)$ is entirely decoupled from that of the variables $\left(A_{1}, A_{2}, B_{1}, B_{2}, Z_{2}\right)$, the attractor of the system (3a)-(3j) will be strange at least for the same values of the parameters $r, \sigma$, and $b$ providing the classical Lorenz system with a chaotic dynamics. See [20,21] for a detailed analysis of the chaotic parameter region of the classical Lorenz system. Additionally, strange attractors could result from choices of the values of $r, \sigma$, and $b$ determining a chaotic dynamics for the $\left(A_{1}, A_{2}, B_{1}, B_{2}, Z_{2}\right)$ variables and a trivial or periodic behavior for the $\left(X_{1}, X_{2}, Y_{1}, Y_{2}, Z_{1}\right)$ variables.

With the "classical" Lorenz parameter values $r=28$, $\sigma=10$, and $b=8 / 3$, the variables $\left(X_{1}, X_{2}, Y_{1}, Y_{2}, Z_{1}\right)$ obviously have an erratic behavior, whereas the variables $\left(A_{1}, A_{2}, B_{1}, B_{2}, Z_{2}\right)$, which describe the faster spatially varying wave components, do not feature any time variability when asymptotic dynamics is considered, as they converge to fixed values $\left(A_{1}^{\infty}, A_{2}^{\infty}, B_{1}^{\infty}, B_{2}^{\infty}, Z_{2}^{\infty}\right)$. As discussed above, the values of $\left(A_{1}^{\infty}, A_{2}^{\infty}, B_{1}^{\infty}, B_{2}^{\infty}\right)$ depend on the initial conditions, whereas, after some algebraic manipulations on Eqs. $(3 \mathrm{e})-(3 \mathrm{~h})$ and $(3 \mathrm{j})$, we obtain $\left(A^{2}\right)^{\infty}=b / 8(r / 2-8),\left(B^{2}\right)^{\infty}$ $=8 b(r / 2-8), Z_{2}^{\infty}=r / 2-8$, and $(A B)^{\infty}=b(r / 2-8)$.

In general, since the fundamental properties of the system do not depend on where in the two-torus the system converges, despite the fact that ergodicity is not obeyed, it makes sense to compute the Lyapunov exponents [2,22] of the system $(3 \mathrm{a})-(3 \mathrm{j})$. In fact, if we compute the Lyapunov exponents $\left(\lambda_{1}, \ldots, \lambda_{10}\right)$ using the algorithm by Benettin et al. [23] with the classical Lorenz parameter values, we obtain 
the same results independently of the initial conditions. The sum of the Lyapunov exponents is given by the trace of the Jacobian $J$ of the system (3a)-(3j) and yields $\operatorname{Tr}(J)=\Sigma \lambda_{j}$ $=-5(2+2 \sigma+b) \approx-123.333$. In particular, we obtain one positive exponent $\left(\lambda_{1} \approx 0.905\right)$, which gives the metric entropy $h=\Sigma_{\lambda_{j}>0} \lambda_{j}$ of the system [2] and matches exactly the same value as the positive Lyapunov exponent in the classical Lorenz system, and three vanishing exponents $\lambda_{2}=\lambda_{3}$ $=\lambda_{4}=0$. The nonhyperbolicity of the system, as described by the presence of two additional vanishing exponents, is related to the existence of two neutral directions due to the symmetry property [Eqs. (4) and (5)]. Moreover, we have six negative Lyapunov exponents, one of which agrees with the negative exponent of the Lorenz system $\left(\lambda_{8} \approx-14.572\right)$. Given the very structure (and derivation) of the model discussed above, the fact that we recover the classical results of the three-component Lorenz system is quite reinsuring. The Kaplan-Yorke dimension [24] of the system is

$$
d_{K Y}=k+\frac{\sum_{j=1}^{k} \lambda_{j}}{\left|\lambda_{k+1}\right|} \approx 4.183,
$$

where $k$ is such that the sum of the first $k$ (4, in our case) Lyapunov exponents is positive and the sum of the first $k$ +1 Lyapunov exponents is negative.

\section{SYMMETRY BREAK AND PHASE MIXING}

Considering a nonvanishing value for Ec amounts to including an additional coupling between the temperature and the streamfunction waves. Such a coupling breaks the symmetry [Eqs. (4) and (5)], so that a dramatic impact on the attractor properties is expected. Using standard multiscale procedure, we can emphasize the emergence of the additional time scale $\mathrm{Ec}^{-1}$. We introduce a slow time variable $\tau$ $=\varepsilon t$ with $\varepsilon=$ Ec. Then we take the expansion $V^{j}(t)=V_{0}^{j}(t, \tau)$ $+\varepsilon V_{1}^{j}(t, \tau)+. .+\varepsilon^{n} V_{n}^{j}(t, \tau)+\ldots$ where $V^{j}, j=1, \ldots, 10$ is a generic variable of the ODEs system considered, we redefine the time derivative $d / d t$ as $d / d t \equiv \partial / \partial t+\varepsilon \partial / \partial \tau$, we plug these new definitions in Eqs. (3a)-(3j), and group together terms with the same power of $\varepsilon$. At zero order-the $\tau$ variable is frozen-we obtain that the variables $V_{0}^{j}$ obey the Eqs. (3a) $-(3 \mathrm{j})$ with $E c=0$. Therefore, for time scales shorter than $O\left(\varepsilon^{-1}\right)$, the symmetries [Eqs. (4) and (5)] described in the previous section are approximately obeyed, which confirms that our multiscale expansion is well-defined.

We hereby consider the specific case where we select the classical values for the other three parameters of the system, so that $r=28, \sigma=10$, and $b=8 / 3$. When large values of Ec are considered $(\mathrm{Ec}>0.045)$, the system loses its chaotic nature as no positive Lyapunov exponents are detected (not shown), whereas periodic motion is realized. The analysis of this transition and of this regime is beyond the scopes of this paper, as we confine ourselves to studying the properties of the system when chaotic motion is realized, thus focusing on the Ec $\rightarrow 0$ limit. We consider Ec $\in[0,0.02]$.

In physical terms, the coupling allows for a mixing of the phases of the thermal and streamfunction waves: after a
$O\left(\varepsilon^{-1}\right)$ time the system "realizes" that the symmetry [Eqs. (4) and (5)] is broken, so that the previously described degeneracies are destroyed and ergodicity is established in the system. Note that, since also in the case of Ec $>0$ the convection does not preferentially act on waves of a specific phase, we have that, pairwise, the statistical properties of $X_{1}$ and $X_{2}$, and of $Y_{1}$ and $-Y_{2}$ are identical and do not depend on the initial conditions. The same applies for the $A$ and $B$ pairs of variables, respectively. If, in particular, we consider the long term averages of the observables $\left\langle X^{2}\right\rangle,\left\langle Y^{2}\right\rangle,\langle X Y\rangle,\left\langle A^{2}\right\rangle$, $\left\langle B^{2}\right\rangle$, and $\langle A B\rangle$, we obtain that in all cases the two addends give the same contribution, e.g., $\left\langle X_{1}^{2}\right\rangle=\left\langle X_{2}^{2}\right\rangle=1 / 2\left\langle X^{2}\right\rangle$. In this case, long is considered with respect to $\varepsilon^{-1}=E c^{-1}$ : these results can be obtained by first- and second-order $\varepsilon$ terms in the multiscale expansion envisioned above. As an example, the $\left\langle X_{1}^{2}\right\rangle=\left\langle X_{2}^{2}\right\rangle=1 / 2\left\langle X^{2}\right\rangle$ identity is obtained by taking the long term average of the first-order $\varepsilon$ term of Eq. (3g).

Whereas having Ec $>0$ is crucial for mixing the phases of waves, the impact of the symmetry break on the long term averages of the physically sensitive observables $O=X^{2}, Y^{2}$, $A^{2}, B^{2}, Z_{1}$, and $Z_{2}$ is relatively weak in the considered Ecvalues range, as we experimentally find that $\Delta \log (\langle O\rangle)$ $\approx$ Ec (not shown). In particular, we obtain that with increasing values of Ec thermal waves $\left(\left\langle Y^{2}\right\rangle,\left\langle B^{2}\right\rangle\right)$ are enhanced, faster spatially varying streamfunction waves $\left(\left\langle A^{2}\right\rangle\right)$ become stronger at the expense of slower spatially varying waves $\left(\left\langle X^{2}\right\rangle\right)$, and finally, the contribution to the convective heat transport due to the faster spatially varying mode $\left(\left\langle Z_{2}\right\rangle\right)$ increases at the expense of the slower mode $\left(\left\langle Z_{1}\right\rangle\right)$. Further investigation on this is surely required.

\section{SYMMETRY BREAK AND HYPERBOLICITY}

We hereby want to take a different point of view on the process of symmetry break of the dynamical system and on its sensitivity with respect to Ec, by analyzing the Ec dependence of the spectrum of the Lyapunov exponents. Again, the classical values for $r, \sigma$, and $b$ are considered. The first, crucial result system is that the system is hyperbolic for any finite positive value of Ec: two of the vanishing Lyapunov exponents branch off from 0 with a distinct linear dependence on Ec, so that $\lambda_{2} \approx 5.1 \mathrm{Ec}$ and $\lambda_{4} \approx-6.0 \mathrm{Ec}$ for $\mathrm{Ec}$ $<0.008$, whereas $\lambda_{3}=0$ corresponds to the direction of the flow. The largest Lyapunov exponent also decreases linearly as $\lambda_{1} \approx 0.905-9.0$ Ec. Time scale separations emerges again, as the two globally unstable directions define two characteristic times $1 / \lambda_{1}$ and $1 / \lambda_{2}$, which are $O(1)$ and $O\left(\mathrm{Ec}^{-1}\right)$, respectively. All the other Lyapunov exponents feature a negligible dependence on Ec, except $\lambda_{8} \approx-14.572+10.0 \mathrm{Ec}$, which ensures that the sum of Lyapunov exponents is independent of Ec. Therefore, we derive that Ec $<0.008$ the metric entropy decreases with Ec as $h(\mathrm{Ec})=\lambda_{1}+\lambda_{2}$ $\approx 0.905-4.0 \mathrm{Ec}$, whereas the Kaplan-Yorke dimension can be approximately written as $d_{K Y}(\mathrm{Ec})=4+\left(\lambda_{1}+\lambda_{2}+\lambda_{3}+\lambda_{4}\right) /\left|\lambda_{5}\right|$ $\approx 4.183-2.0 \mathrm{Ec}$. For larger values of $\mathrm{Ec}$, linearity is not obeyed (except for $\lambda_{2}$ and $\lambda_{4}$ ), whereas a faster, monotonic decrease in both the metric entropy and the Kaplan-Yorke dimensions are found. See Figs. 1(a)-1(c) for results for values of Ec up to 0.02 . We then conclude that, in spite of 

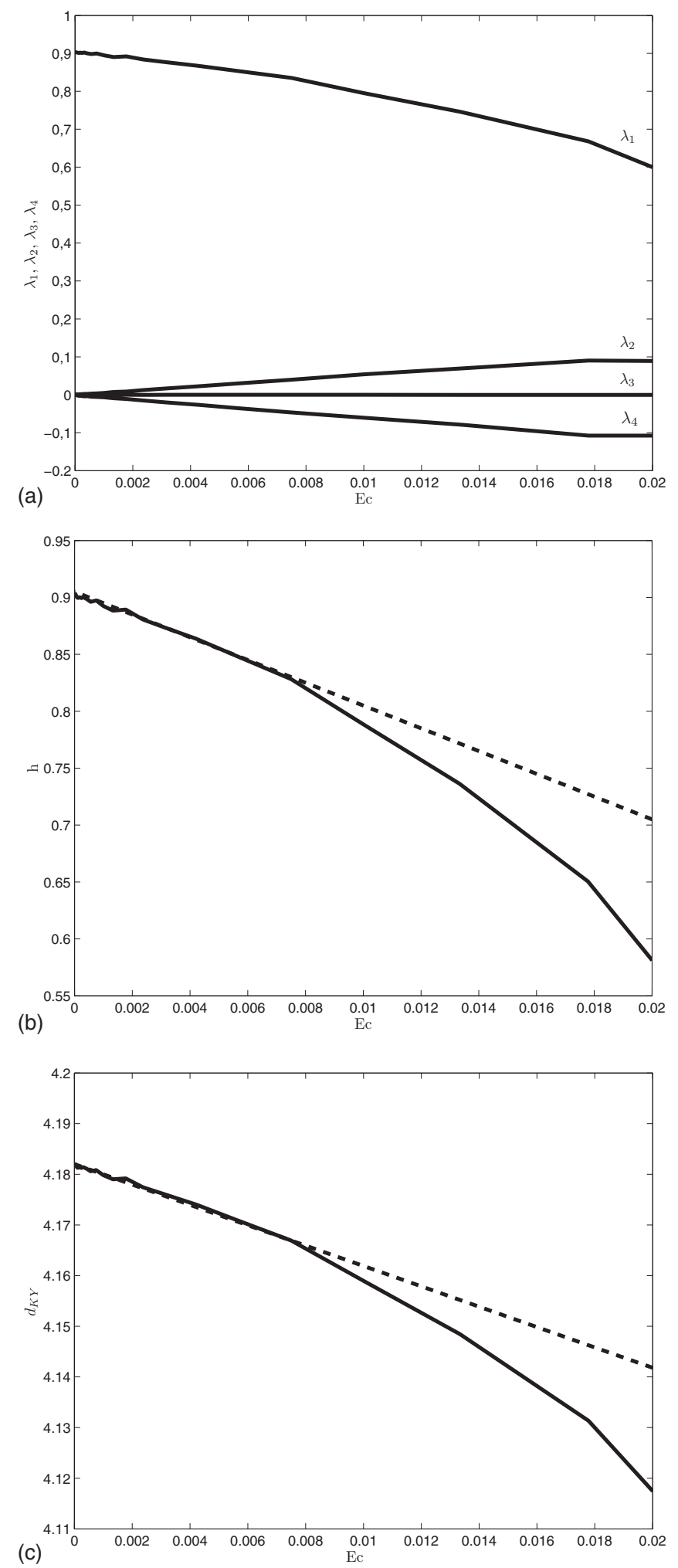

FIG. 1. Four largest Lyapunov exponents (a), metric entropy $h$ (b) and Kaplan-Yorke $d_{K Y}$ dimension (c) as a function of Ec. Note the continuity for $\mathrm{Ec}=0$ of all parameters and the distinct linear behavior - see specifically the dashed lines in (b) and (c) — for Ec $<0.008$. The linear behavior of the second and fourth Lyapunov exponents branching off zero in (a) extends throughout $\mathrm{Ec} \approx 0.018$. Details in the text. introducing a second unstable direction, which is responsible for mixing the phases of the waves, the inclusion of the impact of the viscous dissipation on the thermal energy balance acts with continuity on the dynamical indicators, by reducing the overall instability, increasing the predictability of the system, and by confining the asymptotic dynamics to a more limited (in terms of dimensionality) set.

\section{SYMMETRY-BREAK AND LOW-FREQUENCY VARIABILITY}

We now focus on spectral properties of the system. We first observe that the variables $A_{1}, A_{2}, B_{1}, B_{2}$, and $Z_{2}$ feature mostly ultralow frequency variability. In order to provide some qualitative highlights on these variables, in Fig. 2 we show, from top to bottom, the projection on $A_{1}$ of three typical trajectories for $\mathrm{Ec}=10^{-3}, \mathrm{Ec}=10^{-4}$, and $\mathrm{Ec}=10^{-5}$, respectively. Going from the top via the middle to the bottom panel, the time scale increases by a factor of 10 and 100 , respectively. The striking geometric similarity underlines that the time scales of the dominating variability for the slow variables can be estimated as $O\left(\mathrm{Ec}^{-1}\right)$, in agreement with the multiscale expansion we have proposed. As we take the Ec $\rightarrow 0$ limit, such a time scale goes to infinity, which agrees with the fact that for $\mathrm{Ec}=0$ these variables converge asymptotically to fixed values. Moreover, as $\left\langle A^{2}\right\rangle=\left\langle A_{1}^{2}+A_{2}^{2}\right\rangle$ $\approx b / 8(r / 2-8)=2$ when $\mathrm{Ec}^{-1}$ long-time averages are considered, Fig. 1 suggests that the system switches (on short-time scales) back and forth between $A_{1}$ - and $A_{2}$-dominated dynamics. The same observation are made for the $B_{1}$ and $B_{2}$ pair of variables.

On the other hand, the dynamics of $X_{1}, X_{2}, Y_{1}, Y_{2}$, and $Z_{1}$ is basically controlled by the $O(1)$ time scale $1 / \lambda_{1} \ll 1 / \lambda_{2}$, so that a clear-cut separation between fast and slow variables can be figured out and quantitatively justified. In Fig. 3 we depict, from the top to bottom panel, some trajectories of the fast variable $X_{1}$ for $\mathrm{Ec}=10^{-3}, \mathrm{Ec}=10^{-4}$, and $\mathrm{Ec}=10^{-5}$, respectively. Similarly to Fig. 2, the time scale of the $x$ axis is scaled according to $\mathrm{Ec}^{-1}$. Since $X_{1}$ is a fast variable, the dominating high-frequency variability component is only barely affected by changing Ec, because $\lambda_{1}$ has a weak dependence on $\mathrm{Ec}$, as discussed in the previous section [see also Fig. 1(a)]. Nevertheless, in Fig. 3 we observe an amplitude modulation occurring on a much slower $O\left(\mathrm{Ec}^{-1}\right)$ time scale of the order of $1 / \lambda_{2}$. Note, as in Fig. 2, that such a slow dynamics of the "coarse-grained" $X_{1}$ variable for various values of Ec is statistically similar when the time is scaled according to $\mathrm{Ec}^{-1}$. As in the previous case, the slow amplitude modulation determines the changeovers between extended periods of $X_{1}$ - and $X_{2}$-dominated dynamics, thus ensuring the phase mixing and ergodicity of the system. In the $\mathrm{Ec} \rightarrow 0$ limit, as discussed above, the relative strength of the two phase components of the $(1,1)$ waves is determined by the initial conditions, as mixing requires $\approx \mathrm{Ec}^{-1}$ time units. The same considerations apply for the $Y_{1}$ and $Y_{2}$ pair of variables.

Further insight can be obtained by looking at the spectral properties of the fast and slow variables; some relevant examples of power spectra for $\mathrm{Ec}=10^{-3}, \mathrm{Ec}=10^{-4}$, and $\mathrm{Ec}$ $=10^{-5}$ are depicted in Fig. 4, where we have considered the 

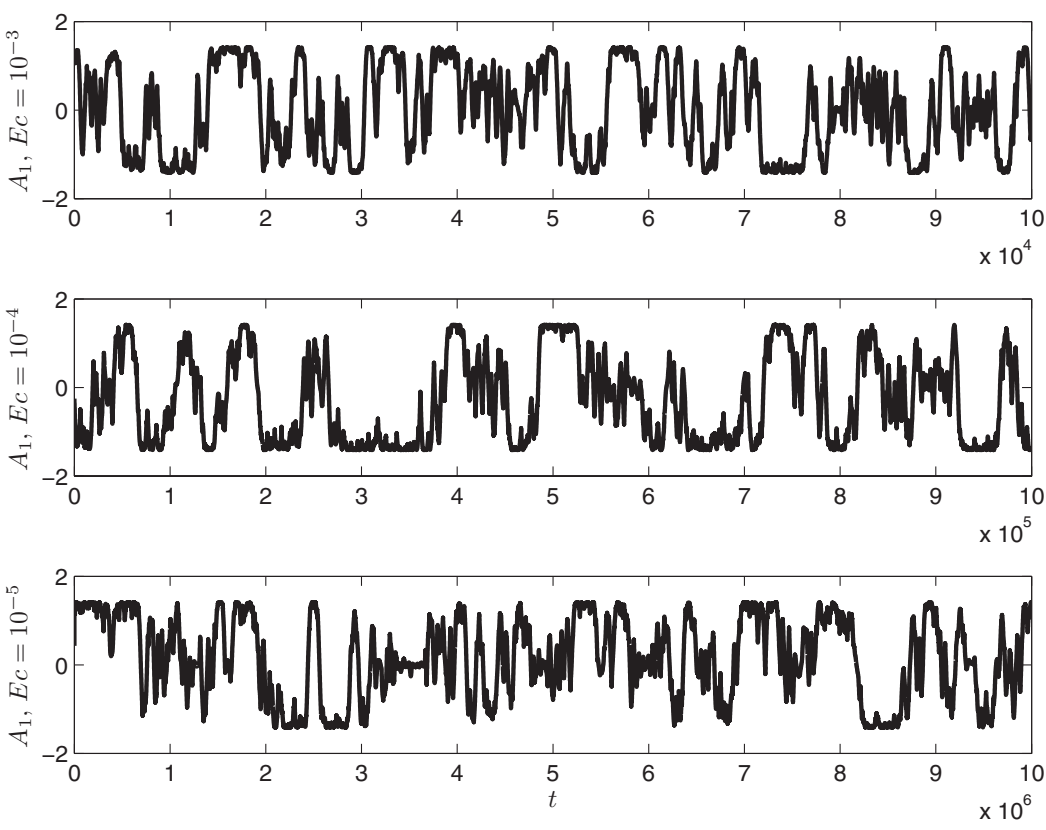

FIG. 2. Impact of the viscous-thermal feedback on the time scales of the system. From top to bottom: typical evolution of the variable $A_{1}$ for different values of the Eckert number $\left(\mathrm{Ec}=10^{-3}\right.$, $\mathrm{Ec}=10^{-4}$, and $\mathrm{Ec}=10^{-5}$, respectively). Note that the time scale is magnified by a factor of 1,10 , and 100 from top to bottom. Details in the text. low-frequency range by concentrating on time scales larger than $10 / \lambda_{1}$. The white-noise nature of the fast variable $X_{1}$ is apparent and so is the overall lack of sensitivity of its spectral properties with respect to Ec. Note that any signature of the amplitude modulation, which is responsible for phase mixing observed in Fig. 3, is virtually absent. This shows how crucial physical processes are masked, due to their weakness, when using a specific metric, such as that provided by the power spectrum. Instead, the slow variable $A_{1}$ has a distinct red power spectrum, which features a dominating $f^{-3 / 2}$ scaling in the low-frequency range (straight line) between the time scales $\approx 0.5 \mathrm{Ec}^{-1}$ and $\approx 10 \mathrm{Ec}^{-1}$. Such a scaling regime dominates the ultralow frequency variability described in Fig. 2 and is responsible for the long-term memory of the signal. For higher frequencies, the spectral density $\rho(P)$ decreases-see the sharp corner in the for $f \approx 0.5 \mathrm{Ec}$ - as $f^{-4}$, and then a classical red noise spectrum $\propto f^{-2}$ is realized for very low values of the spectral density. Note also that, in agreement with our visual perception of Fig. 2, the spectral density $\rho(P)$ scales according to $\mathrm{Ec}^{-1}$ in a large range of energy-containing time scales.

\section{SUMMARY AND CONCLUSIONS}

In this work we have derived and thoroughly analyzed a 10 -variable system of Lorenz-like ODEs obtained by a severe spectral truncation of the 2D $x-z$ convective equations for the streamfunction $\psi$ and temperature $\theta$ in Boussinesq approximation and subsequent nondimensionalization. In the truncation we retain the terms corresponding to the real and imaginary part of the modes $\Psi_{m, n}$ (streamfunction) and $\Theta_{m, n}$ (temperature) associated with the $x-z$ wavevector
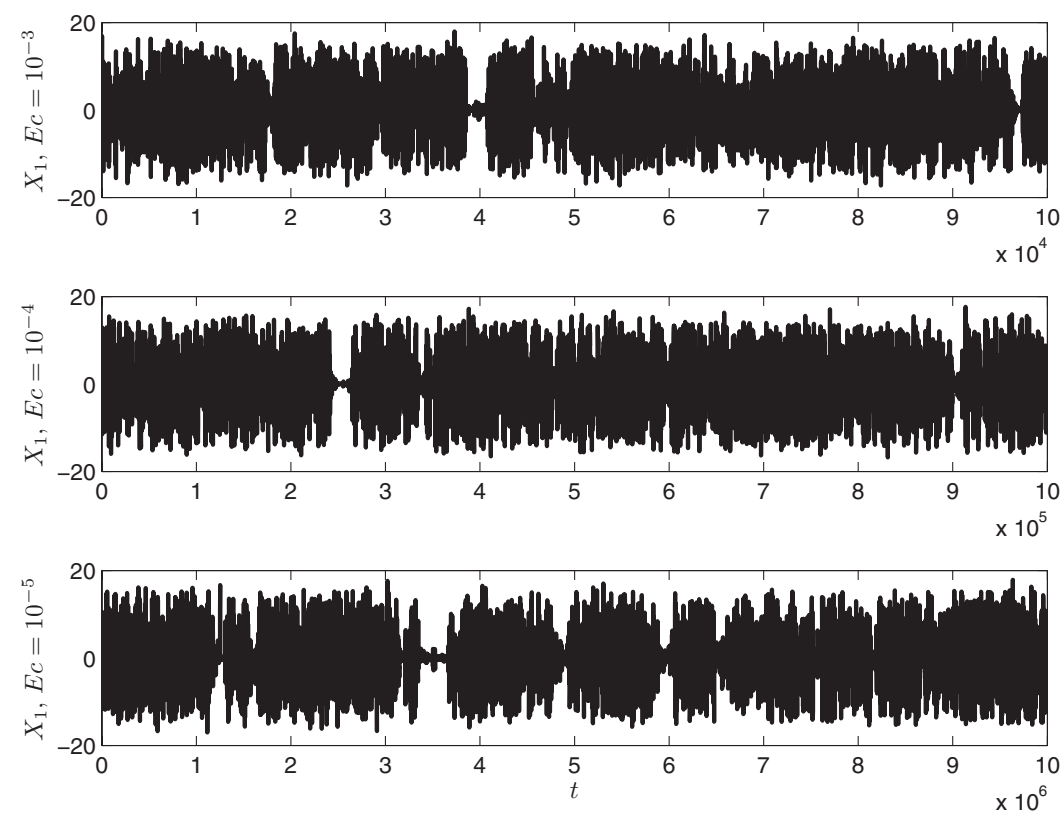

FIG. 3. Impact of the viscous-thermal feedback on the time scales of the system. From top to bottom: typical evolution of the variable $X_{1}$ for different values of the Eckert number $\left(\mathrm{Ec}=10^{-3}\right.$, $E c=10^{-4}$, and $E c=10^{-5}$, respectively). Note that the time scale is magnified by a factor of 1,10 , and 100 from top to bottom. Details in the text. 


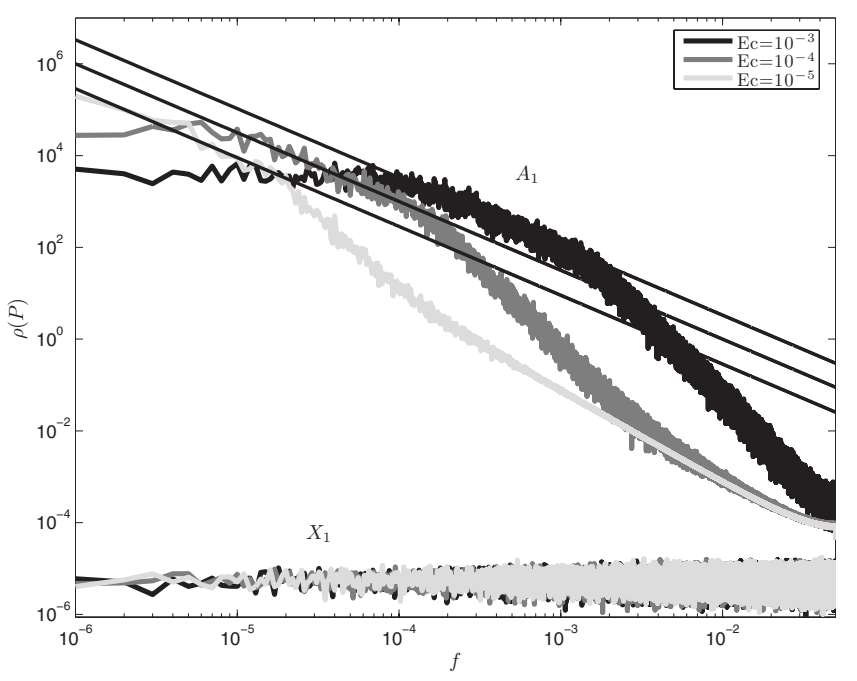

FIG. 4. Power spectrum of $A_{1}$ and $X_{1}$ (multiplied times $10^{-8}$ ) for $\mathrm{Ec}=10^{-3}, \mathrm{Ec}=10^{-4}$, and $\mathrm{Ec}=10^{-5}$ in units of power per unit frequency. The straight lines correspond to $f^{-3 / 2}$ scalings. Details in the text.

$\vec{K}=(\pi a n, \pi m)$ with $(m, n)=(1,1)$ and $(m, n)=(2,2)$, and the imaginary part of the $x$-symmetric modes $\Theta_{0,2}$ and $\Theta_{0,4}$. As modes characterized by a faster varying spatial structure and different parity are added to the Lorenz spectral truncation, which describes the dynamics of the real part of $\Psi_{1,1}$ and of the imaginary part of $\Theta_{1,1}$ and $\Theta_{0,2}$ only, the system we consider in this work represents the thermal impact of viscous dissipation, controlled by the Eckert number Ec. The presence of a fourth parameter-together with the usual Lorenz parameters $r$ (relative Rayleigh number), $\sigma$ (Prandl number), and $b$ (geometric factor) - marks a crucial difference between this ODEs system and other extensions of the Lorenz system proposed in the literature-see, e.g., [4-6]. Moreover, as can be deduced following the argumentations of Nicolis [18], this system specifically allows for the closedform computation of the entropy production, as the Eckert number enters into its evaluation. Up to our knowledge, this is the first ODE system obtained as a truncation of the convective equations presented in the scientific literature featuring all these additional properties.

The following results are worth mentioning:

(1) When the Eckert number is set to 0, as common in most applications, the dynamical system is invariant with respect to the action of a specific symmetry group, which basically shifts the phases of the streamfunction and thermal waves with $m>0$. At a physical level this implies that the initial conditions set the relative strength of waves with the same spatial structure but $\pi / 2$-shifted phases. These results extend the findings presented in [19], where a five-variable system was considered. In particular, it is found that the Lorenz system is included in the ODEs system analyzed here when specific initial conditions, which set the parity of the slower spatially varying modes, are selected. The absence of phase mixing implies a lack of ergodicity, and the degeneracy of the dynamics is reflected also in the nonhyperbolicity of the system. When the classical parameters' values $r$ $=28, \sigma=10$, and $b=8 / 3$ are selected, three of the Lyapunov exponents vanish, one corresponding to the direction of the flow, the other two accounting for the toroidal symmetry. The value of the only positive exponent coincides with that of the Lorenz system, and the value of one of the six negative exponents agrees with that of the negative Lyapunov exponent of the Lorenz system. Therefore, the Lorenz system contains already all the interesting unstable dynamics described by this extended ODEs system, and features exactly the same value for the metric entropy. Correspondingly, while the five variables (fast) describing the modes $\Psi_{1,1}, \Theta_{1,1}$, and $\Theta_{0,2}$ have an erratic behavior, the other five variables (slow) converge to fixed values.

(2) When $E c \neq 0$, the symmetry of the system is broken, and coupling occurs between the fast and slow variable over a time scale $O\left(\mathrm{Ec}^{-1}\right)$. This is clarified by adopting standard multiscale formalism. If we select, as in the original Lorenz system, $r=28, \sigma=10$, and $b=8 / 3$, the system is chaotic for $0<\mathrm{Ec} \leq 0.045$, whereas for higher values of Ec a quasiperiodic regime is realized. In the chaotic regime, the symmetry break is accompanied by the establishment of a hyperbolic dynamics: two Lyapunov exponents branch off from zero (one positive, one negative) linearly with Ec. Therefore, a second unstable direction with a second $O\left(\mathrm{Ec}^{-1}\right)$ time scale $1 / \lambda_{2} \gg 1 / \lambda_{1}$ is established. Overall, the impact of the thermal-viscous feedback is stabilizing, as indicated by the metric entropy and the Kaplan-Yorke attractor dimension monotonically decreasing with increasing Ec, with a marked linear behavior for $\mathrm{Ec} \leq 0.008$.

The coupling establishes dynamics on time scales of the order of $\mathrm{Ec}^{-1}$ responsible for the changeovers between extended periods of dominance of waves of specific phase, both for the slow and for the fast variables. Such dynamical processes, which result from small terms in the evolution equations, correspond to the mixing of phases of the waves and ensure the ergodicity of the system. In particular, the slow variables have a nontrivial time evolution and are characterized by a dominating $f^{-3 / 2}$ scaling in the low-frequency range for time scales between $0.5 \mathrm{Ec}^{-1}$ and $10 \mathrm{Ec}^{-1}$. Instead, in the case of fast variables, the phase mixing appears as a slow amplitude modulation occurring on time scales of $\mathrm{Ec}^{-1}$ which superimposes on the fast dynamics controlled by the $O(1)$ time scale $1 / \lambda_{1}$.

The system presented in this paper features very rich dynamics and, therefore, may have prototypical value for phenomena generic to complex systems, such as the interaction between slow and fast variables and the presence of long term memory. Moreover, analysis shows how, neglecting the coupling of slow and fast variables only on the basis of scale analysis - as usually done when discarding the Eckert number - can be catastrophic. In fact, this leads to spurious invariances that affect essential dynamical properties (ergodicity, hyperbolicity) and that cause the model losing its ability to describe intrinsically multiscale processes. We have shown that a standard multiscale approach allows for understanding the role of the small parameter controlling the coupling. This may suggest that a careful reexamination of the scaling procedures commonly adopted for defining simplified models, especially in the climate science community, may be fruitful in the development of more efficient modeling strategies. Note that in a recent study [25] we have tested 
that refeeding the kinetic energy lost to dissipation as positive thermal forcing to the fluid (which corresponds to considering Ec $>0$ ) brings the long-term global energy budget of the system closer to zero by an order of magnitude.

Whereas the numerical values presented in this paper refer to a specific choice for the parameters $r, \sigma$, and $b$, analogous properties - such as the symmetry break and the ensuing emergence of ergodicity and multiscale processes, the linearity of the Lyapunov exponents with respect to $\mathrm{Ec}^{-1}$, the low-frequency variability are expected for any triplet of $r, \sigma$, and $b$ leading to a chaotic dynamics in the considered system.

We can point at several possible future lines of research:

(1) extensive analysis of the properties of the proposed system for different values of the parameters $r, \sigma$, and $b$, along the lines of the detailed investigation of the phase diagram of the classical Lorenz system presented in [20,21];

(2) extension of the present analysis to higher-order truncation ODEs systems, with detailed investigation of the Ec $=0$ invariance properties, of the impact on these symmetries resulting from setting Ec $>0$, and the ensuing multiscale analysis. This is especially relevant in the context of the results presented by Franceschini and Tebaldi [26] and
Franceschini et al. [27], who emphasized that spectral truncation and modes selections procedures have to be critically addressed.

(3) definition, following [7], of the minimal truncated 3D model of convection able to represent the thermal impact of viscous dissipation;

(4) analysis of the long-term memory of the slow variables and of the related statistical properties of extreme events in terms of the Ec dependence of GEV parameters $[28,29]$;

(5) investigation of how predictability properties of the system depend on Ec: is there a range of values of Ec without exhibiting the unrealistic return of forecast accuracy as shown in several low dimensional systems? (see, e.g., [16];

(6) evaluation of the entropy production of the system, along the lines of Nicolis [18].

\section{ACKNOWLEDGMENTS}

The authors wish to thank C. Penland for intellectual stimulations and an anonymous reviewer for providing constructive criticism.
[1] E. N. Lorenz, J. Atmos. Sci. 20, 130 (1963).

[2] D. Ruelle, Chaotic Evolution and Strange Attractors (Cambridge University Press, Cambridge, 1989).

[3] B. Saltzman, J. Atmos. Sci. 19, 329 (1962).

[4] J. H. Curry, Commun. Math. Phys. 60, 193 (1978).

[5] Z. Wenyi and Y. Peicai, Adv. Atmos. Sci. 3, 289 (1984).

[6] D. Roy and Z. E. Musielak, Chaos, Solitons Fractals 31, 747 (2007).

[7] P. Reiterer, C. Lainscsek, F. Schürrer, C. Letellier, and J. Maquet, J. Phys. A 31, 7121 (1998).

[8] V. Franceschini, J. Stat. Phys. 22, 397 (1980).

[9] V. Franceschini, C. Giberti, and Z. Xheng, Nonlinearity 6, 251 (1993).

[10] C. Sparrow, The Lorenz, Equations. Bifurcations, Chaos, and Strange Attractors (Springer, New York, 1984).

[11] C. Bonatti, L. J. Diaz, and M. Viana, Dynamics Beyond Uniform Hyperbolicity: A Global Geometric and Probabilistic Perspective (Springer, New York, 2005).

[12] V. Lucarini, J. Stat. Phys. 131, 543 (2008).

[13] D. Ruelle, Phys. Lett. A 245, 220 (1998).

[14] D. Ruelle, Nonlinearity 11, 5 (1998).

[15] V. Lucarini, J. Stat. Phys. 134, 381 (2009).

[16] L. A. Smith, C. Ziehmann, and K. Fraedrich, Q. J. R. Meteorol.
Soc. 125, 2855 (1999).

[17] T. N. Palmer, Bull. Am. Meteorol. Soc. 74, 49 (1993).

[18] C. Nicolis, Q. J. R. Meteorol. Soc. 125, 1859 (1999).

[19] Z.-M. Chen and W. G. Price, Chaos, Solitons Fractals 28, 571 (2006).

[20] R. Barrio and S. Serrano, Physica D 229, 43 (2007).

[21] R. Barrio and S. Serrano, Physica D (to be published).

[22] V. Oseledec, Trans. Mosc. Math. Soc. 19, 197 (1968).

[23] G. Benettin, L. Galgani, A. Giorgilli, and J. M. Strelcyn, Meccanica 15, 9 (1980).

[24] J. L. Kaplan and J. A. Yorke, in Functional Differential Equations and Approximations of Fixed Points, edited by H.-O. Peitgen and H.-O. Walter (Springer, Berlin, 1979).

[25] V. Lucarini, K. Fraedrich, and F. Lunkeit, e-print arXiv:0905.3669.

[26] V. Franceschini and C. Tebaldi, Meccanica 20, 207 (1985).

[27] V. Franceschini, C. Giberti, and M. Nicolini, J. Stat. Phys. 50, 879 (1988).

[28] S. Coles, An Introduction to Statistical Modeling of Estreme Values (Springer, Heidelbeg, 2001).

[29] M. Felici, R. Vitolo, V. Lucarini, and A. Speranza, J. Atmos. Sci. 64, 2137 (2007). 